\title{
Miliary pulmonary tuberculosis after the first dose of intravesical BCG instillation in a patient with high-grade bladder cancer
}

\author{
Adam Stępień', Michał Brudło', Tomasz Stachura ${ }^{2}$, Paulina Marcinek ${ }^{3}$, \\ Jerzy Soja', Krzysztof Stadek ${ }^{2}$ \\ 'Student Scientific Group of Interventional Pulmonology, Jagiellonian University Medical College, Krakow, Poland \\ ${ }^{2}$ Department of Pulmonology and Allergology, Jagiellonian University Medical College, Krakow, Poland \\ ${ }^{3}$ Department of Radiology, Jagiellonian University Medical College, Krakow, Poland
}

A 62 years old patient, who was diagnosed with high-grade T1 (T1HG) bladder cancer, after transurethral resection (TURB) (February 2018) and re-TURB (March 2018), with a history of diabetes mellitus type 2, hypertension, dyslipidemia, coronary artery disease and three percutaneous coronary interventions was admitted to the Urology Department. On admission he presented features of acute liver failure and right ventricular heart failure, accompanied by high fever, general malaise and elevated inflammatory response markers, which occurred after the first intravesical Bacillus Calmette-Guérin (BCG) instillation. The immunotherapy was started two weeks before hospitalization. Computed tomography (CT) revealed hepato- and splenomegaly, a small amount of fluid in the peritoneal cavity and slightly thickened wall of the bladder ( $7 \mathrm{~mm}$ ).

The patient was relocated to the Pulmonology Department in serious general condition, presenting features of respiratory failure, decompensated heart failure and hectic fever up to $39^{\circ} \mathrm{C}$. On physical examination bibasal crackles, hepatomegaly and peripheral oedema were found. Moreover, elevated values of C-reactive protein $(109.2 \mathrm{mg} / \mathrm{L})$, procalcitonine $(0.87 \mathrm{ng} / \mathrm{mL})$, alanine aminotransferase $(104 \mathrm{U} / \mathrm{L})$, aspartate aminotransferase (124 U/L), alkaline phosphatase (823 U/L) and gamma-glutamyl transpeptidase (1130 U/L) were detected. A bronchoalveolar lavage (BAL) was performed twice to take samples for cytologic, bacteriological and mycological tests as well as Bactec and ProbeTecET genetic probes. Moreover, bronchofiberoscopy with endobronchial ultrasound-guided transbronchial needle aspiration (EBUS-TBNA) of subcarinal lymph nodes did not point towards a proliferative disease. In addition, blood and urine culture, diagnostic tests for infectious diseases particularly for HIV, were carried out. The BAL findings were positive for Mycobacterium tuberculosis complex in genetic testing. Other microbiological tests were negative. A chest CT scan pointed to multiple lung changes which composed a picture of miliary pulmonary tuberculosis with concomitant oedematous lesions (Figure 1A).

The patient was treated with passive oxygen therapy, broad-spectrum antibiotics (meropenem, levofloxacin and vancomycin), itraconazole and systemic corticosteroids. The first intensive phase of anti-tuberculosis treatment targeting Mycobacterium bovis was started with isoniazid (INH), rifampicin (RMP) and ethambutol (EMB). As a result, a significant improvement of general condition was observed with resolution of fever and normalization of laboratory results. The corticosteroid treatment has been continued in decreasing doses for 3 months, whereas anti-tuberculosis regimens for 6 months (INH, RMP, EMB treatment for 2 months followed by continuation phase of INH and RMP for 4 months). In a control chest CT near complete remission of pulmonary changes was stated (Figure 1B).

Currently, BCG immunotherapy is considered as the gold standard treatment for non-muscle invasive bladder cancer at high risk of progression or recurrence [1]. Progression-free survival at three years observation is $86.8 \%$ and results are better in comparison to local chemotherapy [2, 3]. Nevertheless, the BCG immunotherapy could be associated with the wide spectrum of adverse effects like dysuria, pyrexia, hematuria or fatigue in $69.5-91 \%$ of cases [4]. The pulmonary involvement is observed only in $0.3-0.7 \%$ of treated patients [4]. Our literature search yielded 35 similar case reports published so far. Moreover, among them only 1 occurred after the first dose of BCG instillation as in our report [5].

Address for correspondence: Adam Stępień, Student Scientific Group of Interventional Pulmonology, Jagiellonian University Medical College, Krakow, Poland e-mail: astepien@poczta.fm

DOI: 10.5603/ARM.a2020.0150

Received: 03.09.2020

Copyright (C) 2020 PTChP

ISSN 2451-4934

Conflict of interest: None declared 

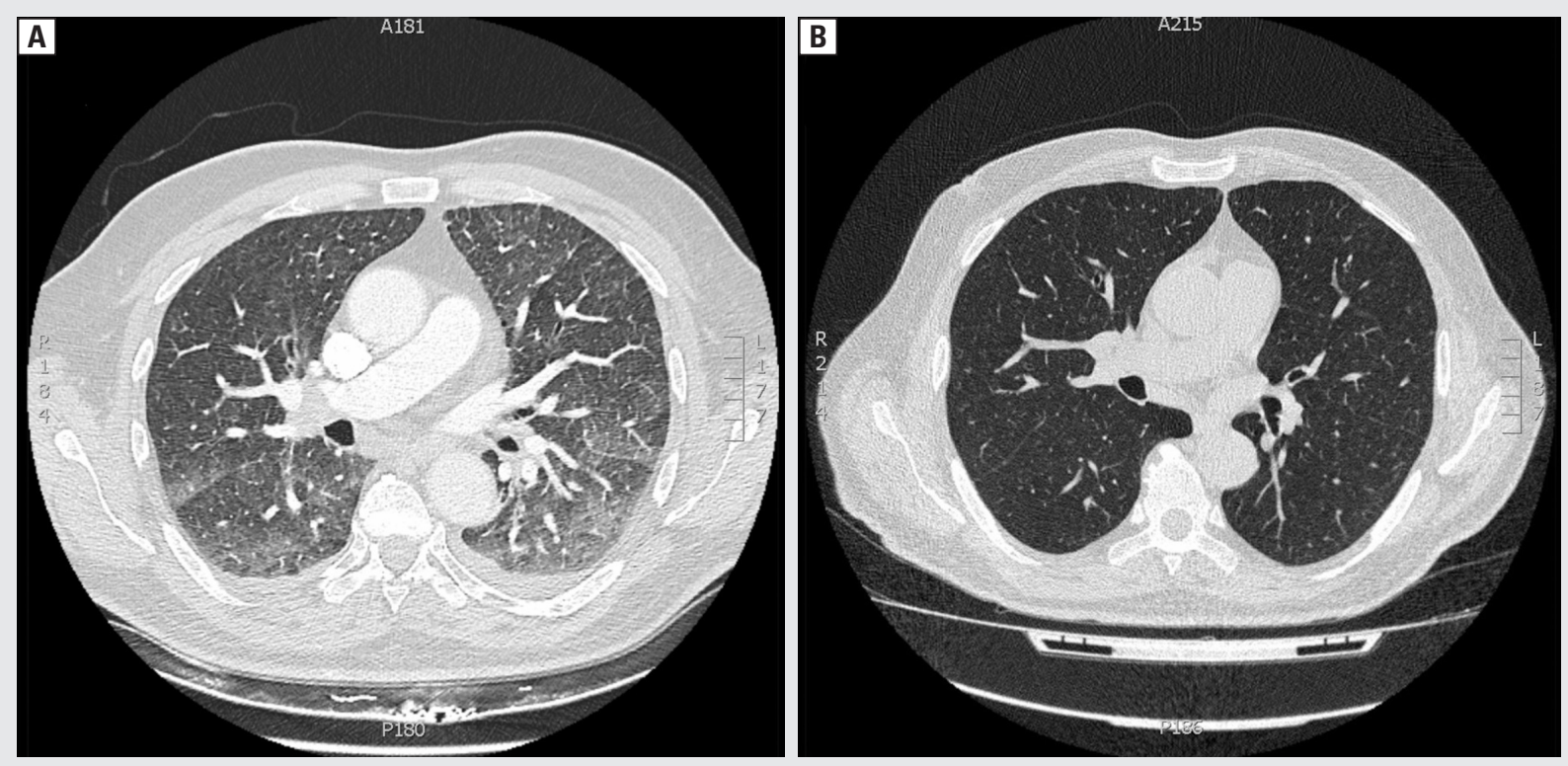

Figure 1A. The chest computed tomography (CT) is indicative of lung micronodular lesions $(0.5-1 \mathrm{~mm})$ accompanied by oedematous changes. Small pleural effusion in the both pleural cavities can be seen. Bilaterally, particularly in the dorsal segments of the lungs, alveolar consolidation and signs of septal thickening are present. B. In comparison to the previous chest CT, after 2 months of treatment, there has been complete resolution of fluid in the pleural cavities. Also noticeable is a nearly complete resolution of lung micronodules with only few up to $0.5 \mathrm{~mm}$ in diameter still present

\section{References:}

1. Pettenati C, Ingersoll MA. Mechanisms of BCG immunotherapy and its outlook for bladder cancer. Nat Rev Urol. 2018; 15(10): 615-625, doi: 10.1038/s41585-018-0055-4, indexed in Pubmed: 29991725.

2. Pirzada MT, Ghauri R, Ahmed MJ, et al. Outcomes of BCG induction in high-risk non-muscle-invasive bladder cancer patients (NMIBC): a retrospective cohort study. Cureus. 2017; 9(1): e957, doi: 10.7759/cureus.957, indexed in Pubmed: 28168135.

3. Shelley MD, Wilt TJ, Court J, et al. Intravesical bacillus Calmette-Guérin is superior to mitomycin C in reducing tumour recurrence in high-risk superficial bladder cancer: a meta-analysis of randomized trials. BJU Int. 2004; 93(4): 485-490, doi: 10.1111/j. 1464-410x.2003.04655.x, indexed in Pubmed: 15008714.

4. G. Calleris, G. Marra, S. Corcione, et. al. Miliary pulmonary infection after BCG intravesical instillation: a rare, misdiagnosed and mistreated complication. Infez Med. 2017; 25: 366-370.

5. To U, Kim J, Chia D. Disseminated BCG: complications of intravesical bladder cancer treatment. Case Rep Med. 2014; 2014: 362845, doi: 10.1155/2014/362845, indexed in Pubmed: 25024707. 